\title{
Creatine Kinase MB Measurement
}

National Cancer Institute

\section{Source}

National Cancer Institute. Creatine Kinase MB Measurement. NCI Thesaurus. Code C64491.

A quantitative measurement of the amount of heterozygous MB-type creatine kinase present in a sample. 